\title{
The System of Knowledge Control Based on Adaptive Semantic Models
}

\author{
Tamara Shikhnabieva ${ }^{1, a *}$ \\ 1 Plekhanov Russian University of Economics, 117997, 36 Stremyanny pereulok, Moscow, \\ Russia \\ ashetoma@mail.ru \\ ${ }^{*}$ Corresponding author
}

Keywords: training, knowledge management, intelligent tutoring systems, adaptive semantic model

\begin{abstract}
The existing automated systems of training and knowledge control have a rigid structure, do not fully take into account students' individual characteristics, i.e. are not non-adaptive. To build adaptive learning systems, along with knowledge about the subject, it is necessary to have some information about knowledge and goals of students (a user model). Considering a user model allows developing adaptive learning systems that identify the level of knowledge of students and, accordingly, provide each user with an individual learning path and an individual electronic textbook. The article presents one of the options for building adaptive learning systems and knowledge control based on the use of achievements of Cybernetics, synergetics, and the theory of artificial intelligence.
\end{abstract}

\section{Introduction}

Currently, there are many practical implementations of automated testing systems, both in individual disciplines, as well as there are universal knowledge assessment systems that are fully or partially invariant to specific disciplines and allow teachers to fill them with information [1,2]. Analysis of the effectiveness of automated testing systems shows that they have a number of drawbacks. The most significant drawbacks of modern approaches to automated testing are:

- The need to formulate answers to the test tasks on the principle of " $\mathrm{A}$ is absolutely correct" - " $\mathrm{B}$ is absolutely wrong". This approach does not allow to organize full testing in poorly formalized disciplines, which are characterized by the dialectical nature of knowledge;

- Primitiveness and inflexibility of procedures for calculating the final grade, reduced either to determining the ratio of the number of correct answers to the number of questions asked, or to the summation of points assigned for each correct answer;

- Impossibility to automate various methods of knowledge control widely used in pedagogical practice (assessment of breadth or depth of knowledge, consideration of the relative importance of certain topics or sections of the discipline under study, choice of test complexity taking into account the level of preparedness and self-esteem of the subject, stimulation of correct answers, etc.);

- Significant labor-intensiveness of manually forming such a multitude of test tasks and answer options for each of them, which can eliminate or minimize the likelihood that the same task would be presented to the test during parallel testing of their knowledge.

Particularly brightly indicated shortcomings of automated testing are manifested in the control of knowledge in the disciplines of the humanitarian, socio-economic, and socio-political cycles. The degree of formalization of knowledge in these disciplines is, by the virtue of dialecticity, too low for their availability to be determined by how well we remember individual facts under examination, precise definitions or specific formulas and rules for their application.

If we proceed from the need to improve the efficiency of any educational process and the use of modern information technologies in education, then the most promising and expedient is the automation of 
the process of pedagogical testing [3-5]. The high degree of formalization and unification of the testing procedure, the possibility of simultaneous testing on several computers, as well as the possibility of organizing remote testing through a local computer network or through the global information network "Internet" predetermined the general interest in this method of knowledge assessment.

It can be argued that the procedures of "classical" testing, based on the paradigm of "one absolutely correct answer, several absolutely wrong answers" and the conclusion of a final assessment from the ratio of correct answers to the questions asked, are inadequate to the majority of teachers with respect to the knowledge assessment process. For many disciplines, knowledge is fundamentally fuzzy in nature and cannot be reduced to unambiguous formulations (for example, disciplines of the humanitarian or social cycles), they generally turn out to be inapplicable.

Consequently, a knowledge control system will be recognized by specific teachers as an effective tool for intermediate or final knowledge control only if it will: a) contain an information model of the subject area relevant to the subject knowledge of a test organizer during particular monitoring period; $b$ ) have the ability to take into account incomplete or not entirely accurate answers; c) contain an adaptive and teachercontrolled procedure for identifying knowledge, analyzing its depth and quality by subsequent reconstruction on this basis of the student's information model; d) derive a final assessment of the student's knowledge based on the comparison of the reference model contained in the automated knowledge control system with the reconstructed model built according to the student's answers.

Building such a system of knowledge control requires the use of fundamentally different approaches to the representation and processing of knowledge, which our article is dedicated to [6].

\section{Methodology}

The system of learning and knowledge control we propose is based on a logical-semantic approach, the principles of constructing artificial intelligence systems $[7,8,9]$ and combines a procedural and declarative approach to the representation of knowledge, which is based on the theory of semantic networks and efficient algorithms.

As is known, "the graph is a very characteristic mathematical object of adaptation" [10]. Therefore, we chose adaptive semantic models as a model of the logical structure of educational material. The adaptive semantic model (ASM) of educational material is understood as a multi-level hierarchical structure in the form of a semantic network represented by a directed graph, at the vertices of which are the concepts of the subject area under study, and the edges indicate the relationship between them [11]. The advantages of the learning process model we propose are particularly significant when controlling the knowledge of students $[11,12]$.

As the study of electronic educational tools used in training shows, many of the existing e-courses are closed systems with rigid models that do not always allow to adapt to a particular level of knowledge of a student [13].

The traditional system of education at different levels tends to give students as much actual material as possible. With this approach, the quality of knowledge is assessed by taking into account the number of facts (concepts, knowledge elements) that a learner operates with and the accuracy of their reproduction. Since the studied concepts of the domain are interconnected and follow each other, relations between such concepts and logical inference rules of specific concepts from more general categories of the subject domain remain aside. This kind of training leads to the formalism of knowledge. Accounting for links and sequence of elements of educational material is especially important when learning on the basis of computer technology.

The semantic network implies the meaning processing of information by a computer, which is necessary when processing students' responses. When controlling knowledge, it is necessary to build a semantic network on the computer screen using pre-known domain concepts, and then students' knowledge model is compared with the model in the database on the topic being searched for. As a result, student's knowledge is monitored. The knowledge control method developed by us also allows structuring questions and creating adaptive tests [14]. 


\section{Results}

On the basis of the proposed approaches, we have developed an intellectual system of training and knowledge control in certain areas of computer science and mathematics, which is used in the educational process of a number of universities. The intelligent learning system consists of a learning module, a web editor, and a knowledge control [13].

The intelligent learning system developed by us automatically generates control tasks of varying complexity, takes into account history of a particular user and is invariant with respect to educational subjects.

Figure 1 presents an example of a control task (network of inquiry) in the form of an adaptive semantic model on the theme "Hierarchical structures."

Some vertices and ACM links are not signed. A learner needs to determine the purpose and connections between the vertices of educational material, selecting them from the list provided.

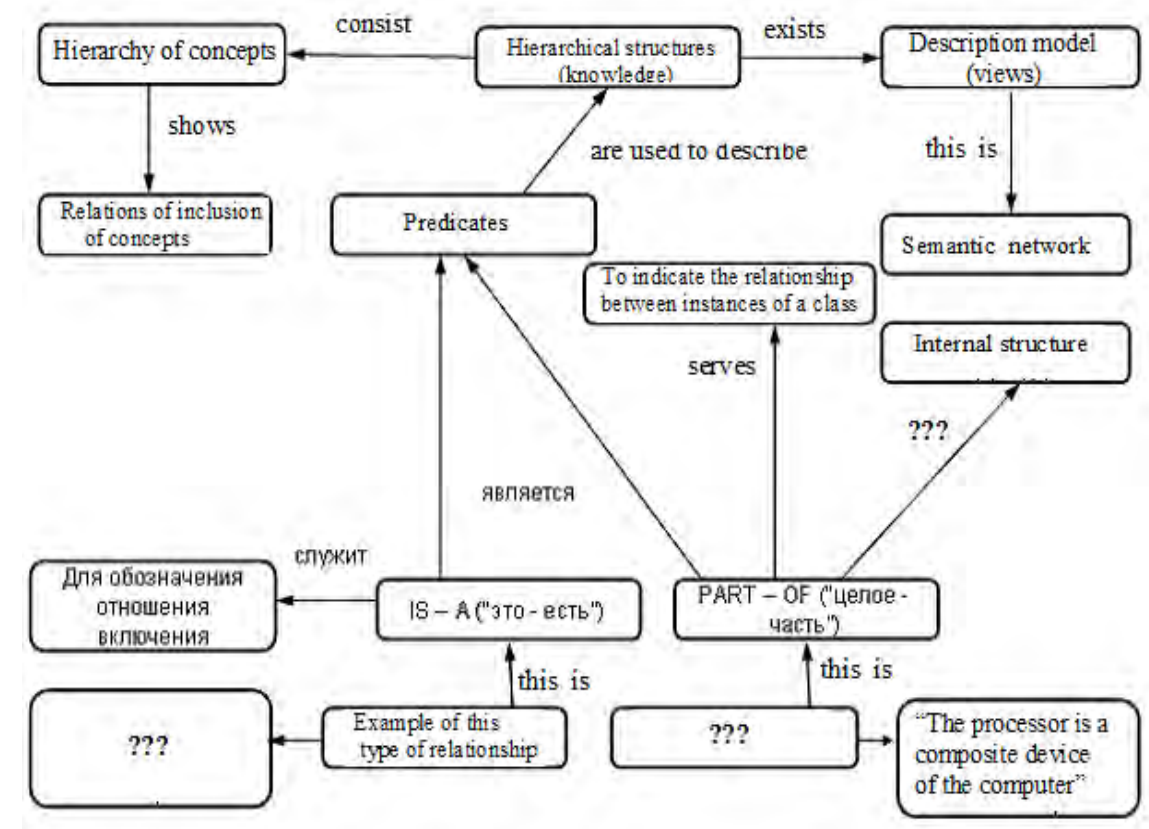

Fig. 1. The control task on the topic "Hierarchical structures."

It should be noted that the process of developing semantic models contributes to the effective acquisition of knowledge. Therefore, training can be conducted not only according to the developed ASM teacher, but also it is possible to give students assignments for their development, which contributes to a better mastery of educational material.

It is necessary to emphasize that the adaptive semantic model implies the semantic processing of information by a computer, which is necessary when processing students' responses. In the process of building semantic models on a computer, students must analyze their own knowledge, which helps them incorporate new knowledge into the structure of existing knowledge $[5,14]$. The result of this training is the effective use of acquired knowledge.

The use of the proposed methodology for the control of knowledge in the form of distance learning [15] also allows for the provision of the following: (a) an individual learning pace when implementing feedback; (b) an activity approach in choosing the solution of the problem, taking into account educational situations; (c) the connection of new concepts with existing concepts and concepts, which improves understanding; (d) the implementation of deep knowledge processing, which increases the use of acquired knowledge in new situations. You can also use the query network to control students' knowledge.

With this form of knowledge control, a student complements the top of the network with relevant concepts from the domain, which are indicated by a question mark and indicate the types of connections between them. Genuine activation of students' cognitive activity is possible only with an expedient sequence of presentation of cognitive tasks, as well as taking into account the characteristics of all elements 
of the tasks being completed. Therefore, along with the structuring of theoretical knowledge, we conducted a classification of educational tasks in computer science

\section{Conclusion}

Thus, the use of adaptive semantic models in modeling a learning process allows us to provide: (a) an individual learning rate during the implementation of feedback; (b) an activity approach in choosing a solution to a problem, taking into account educational situations; (c) a connection of new concepts with existing concepts, which improves understanding; and (d) implementation of deep knowledge processing, which increases the ability to apply knowledge in new situations.

The advantages of the model of knowledge representation offered by us are especially significant when controlling the knowledge of students. The knowledge control method developed by us also allows us to structure questions and create adaptive tests.

Such an organization of knowledge control contributes to high-quality learning, since students analyze the basic structure of the concepts being studied, associating new concepts with them.

\section{Acknowledgments}

This research was performed in the framework of the basic part of the scientific research state task in the field of scientific activity of the Ministry of Education and Science of the Russian Federation, project "Intellectual analysis of big textual data in finance, business, and education by means of adaptive semantic models," Grant no. 2.9577.2017/8.9.

\section{References}

[1] Strogalov, A. S. (1998). Computer tutoring systems: some problems of their development: university training in the information society (pp. 68-72). Moscow, Russia: RGGU.

[2] Strogalov, A. S., \& Shekhovtsov, S. G. (1998). Thinking, language and intellectual education. Intellectual systems, 3(3-4), pp. 5-50.

[3] Dikarev, S. B., Gura, V. V., \& Tselykh, A. N. (2006). Bulletin of Computer and Information Technologies, 5, 24-35.

[4] Dikarev, S. B. (2004). Perspective Information Technologies and Systems (ITPAS), 11, 13-19.

[5] Jonassen, D. (1996). Computer Science and Education, 4.

[6] Uskov, V. L., \& Uskov, A. V (2007). Advanced Technology for Learning, 4(3), pp. 45-56.

[7] Gavrilova, T. A., \& Khoroshevsky, V. F. (2000). Knowledge bases of intellectual systems. S. Petersburg, Russia: Peter.

[8] Grigoriev, A. D., \& Lande, D. V. (2005). Adaptive interface for refining requests to the content monitoring system InfoStream. Proceedings of the international seminar "Dialogue 2005" (pp. 109111). Russia.

[9] Russell, S., Norvig, P. (2006). Artificial intelligence: a modern approach (2nd ed.). Moscow: Williams Publishing House.

[10] Rastrigin, L. A. (1981). Adaptation of complex systems. Riga, USSR: Zinatne.

[11] Shikhnabiyeva, T. Sh. (2009). Methodological foundations of the representation and control of knowledge in the field of informatics using adaptive semantic models (Doctoral Dissertation). Moscow, Russia.

[12] Shikhnabiyeva, T. Sh. (2016). Intellectual training and knowledge control system based on adaptive semantic models. Proceedings of the VI International Scientific and Technical Conference "Open Semantic Technologies of Intelligent Systems Design" Open Semantic Technologies for Intelligent 
Systems OSTIS-2016 (pp. 503-506). Minsk, Belarus: BSUIR.

[13] Starichenko, B. E., Semenova, I. N., \& Slepukhin, A. V. (2014). Education and Science, 9(118), 123132.

[14] Shikhnabieva, T., \& Beshenkov, S. (2016). Intelligent System of Training and Control of Knowledge, Based on Adaptive Semantic Models. Smart Innovation, System and Technologies, 99, pp. 84-93.

[15] Shikhnabieva, T., Brezhnev, A., Saidakhmedova, M., Brezhneva, A., \& Khachaturova, S. (2018). Intellectualisation of educational information systems based on adaptive semantic models. 4rd international kes conference on smart education and e-learning/kes-seel-18, 20-22 June 2018. Gold Coast, Australia. 